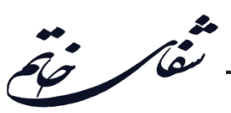

\title{
The Effect of Intracerebroventricular Administration of Streptozocin on Cell Proliferation in Subventricular Zone Stem Cells in a Rat Model of Alzheimer's Disease
}

\author{
Hoda Pasand Mozhdeh", ${ }^{1,}$ Bahman Zeynali', Hadi Aligholi², ${ }^{1}$, Iraj Kashani Radgerdi ${ }^{4}$, Sajad Sahab Negah², \\ Gholamreza Hassanzadeh $^{4 *}$ \\ ${ }^{1}$ Department of Biology, Faculty of Sciences, University of Tehran, Tehran, Iran \\ ${ }^{2}$ Shefa Neuroscience Research Center, Khatam Alanbia Hospital, Tehran, Iran \\ ${ }^{3}$ Department of Neuroscience, School of Advanced Medical Sciences and Technologies, Shiraz University of Medical Sciences, \\ Shiraz, Iran \\ ${ }^{4}$ Department of Anatomy, School of Medicine, Tehran University of Medical Sciences, Tehran, Iran
}

\section{A BSTRACT}

Introduction: Alzheimer's disease (AD) is the most common neurodegenerative disorder with the prevalence of one eighth or about $10 \%$ of people older than 65 years old. Aiming to induce progressive dementia and neurodegeneration, intracerebroventricularly (ICV) injection of streptozocin (STZ), a diabetogenic factor, was performed. Since this model leads to behavioral disorders, impaired energy, and glucose utilization, it is an appropriate model for AD. Reduction of the potency of stem cells in the subventricular zone (SVZ), one of the brain neurogenic regions, in $\mathrm{AD}$ has been reported. In this study, we examined the effect of ICV administration of STZ on stem cell proliferation in SVZ. Materials and Methods: Thirty male Wistar rats were classified into three groups, including control, sham, and STZ. Drug injection ( $3 \mathrm{mg} / \mathrm{kg}$ - ICV) was performed in lateral ventricles in days 1 and 3 after recovery of operation. Finally, after SVZ cell culture, cell proliferation was measured by evaluation of morphology, number, and diameter indexes of neurospheres. Results: Our study revealed that proliferation indexes, such as diameter and the number of neurosphere, were significantly decreased in the treatment group compared to control and sham groups. Conclusion: Our data has shown that application of STZ decreases proliferation of stem cells in SVZ of the brain.

\section{Key words:}

1. Alzheimer Disease

2. Streptozocin

3. Neural Stem Cells

* Corresponding Author: Gholamreza Hassanzadeh

E-mail: hassanzadeh@tums.ac.ir 


\title{
اثر تجويز درون بطنى استريتوزوسين بر تكثير سلول در سلولهاى بنيادى ناحيةٌ تحت بطنى در يك مدل موش صحر ايى مبتلا به بيمارى آلزايمر
}

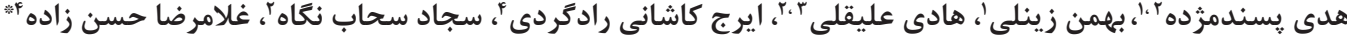 \\ 'كروه زيست شناسى، دانشكده علوم، دانشاه تهران، تهران، ايران \\ 'مركز تحقيقات علوم اعصاب شفا، بيمارستان خاتم الانبياء، تهران، ايران \\ "َّروه علوم اعصاب، دانشكده فناورىهاى نوين يزشكى، دانشكاه علوم يزشكى شيراز، شيراز، ايران \\ "
}

كليد وازهها:

ا ب. بيمارى آلزايمر

r. ب. استر يتوزوسين

ب.

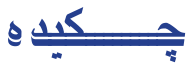

مقدمه: بيمارى آلزايمر شايعترين اختلال تحليل برنده عصبى با شيوع يك هشتم يا حدود ده

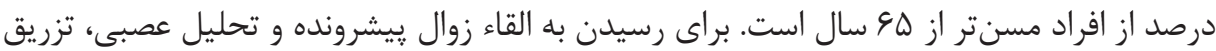

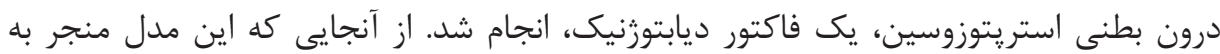

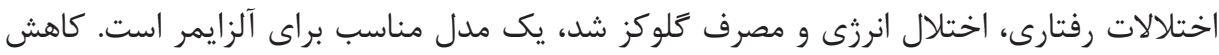

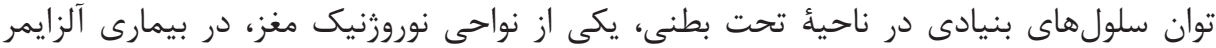

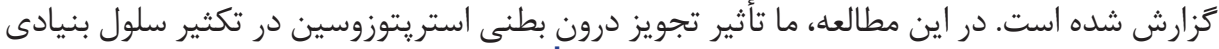

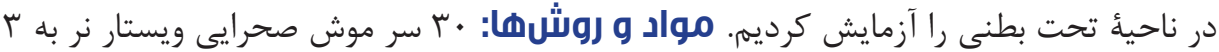

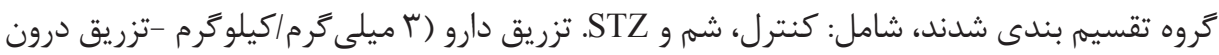

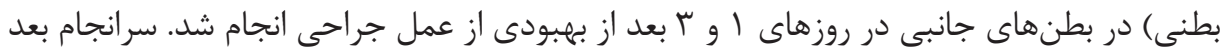

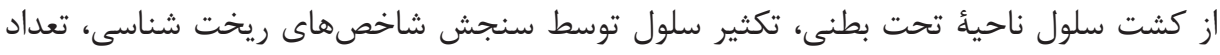

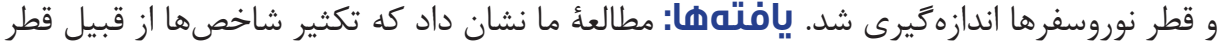

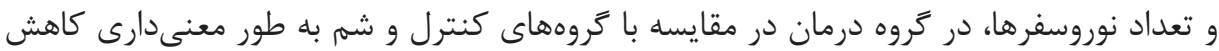

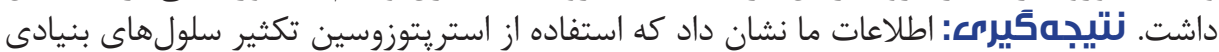
در ناحيةُ تحت بطنى مغز را كاهش مى دهد. 


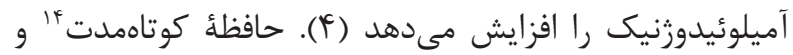

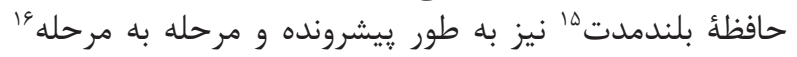

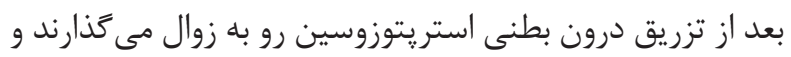

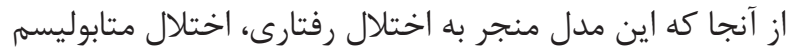

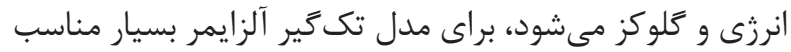

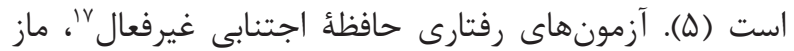

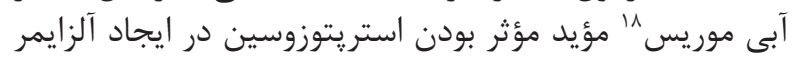

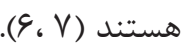

سالهاى بسيارى عقيده بر اين بود كه سيستم عصبى مركزِى

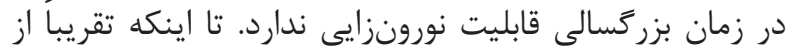

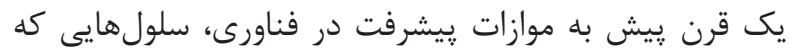

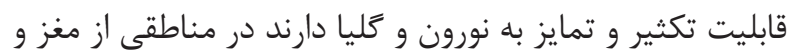

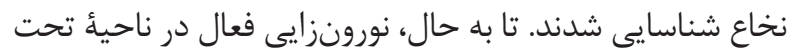

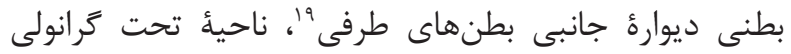

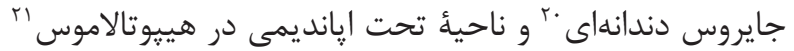

مشخص شده است (1) - (1).

در اوايل قرن حاضر Allen، سلولهاى ميتوزى را در ناحئ

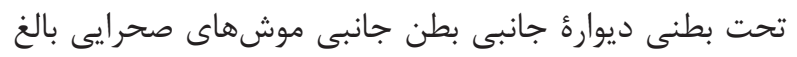

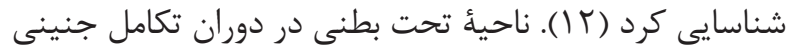

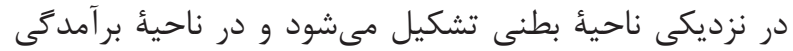

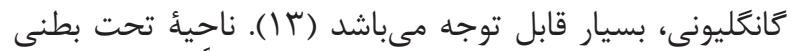

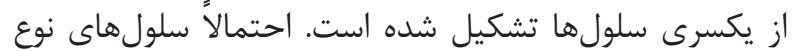

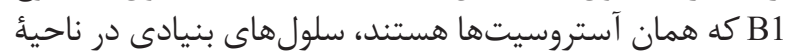

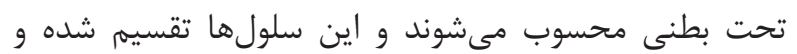

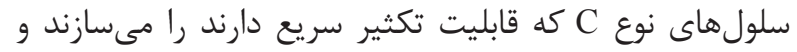

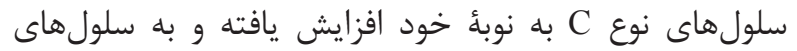

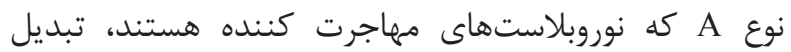

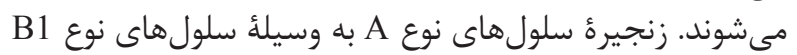

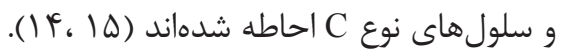

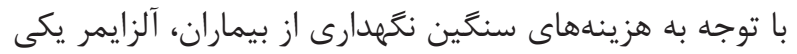

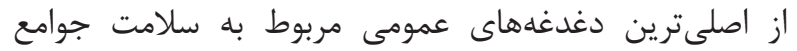

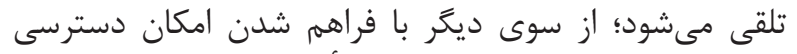

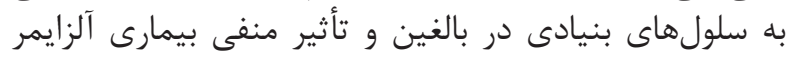

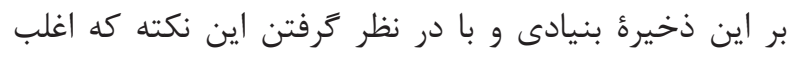

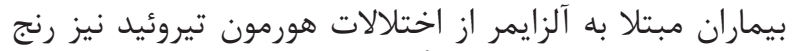

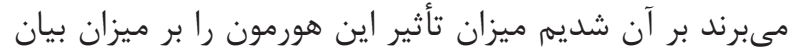

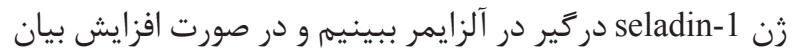
آن، نقش محافظتى اين هورمون در مقابل بيمارى آلزايمر ثابت دابت بـان

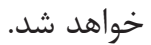

\footnotetext{
${ }^{1}$ Alzheimer's disease

${ }^{2}$ Neurodegenerative

${ }^{3}$ Alois Alzheimer

${ }^{4}$ Atrophy

${ }^{5}$ Amyloid precursor protein $(\mathrm{A} \beta)$

${ }^{6}$ Neurofibrillary tangle

${ }^{7}$ Familial Alzheimer's disease

${ }^{8}$ Sporadic Alzheimer disease

${ }^{9}$ Intracerebroventricular

${ }^{10}$ Streptozotocin

${ }^{11}$ Neuromodulatory
}

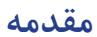

بيمارى آلزايمر' شايعترين اختلال تحليلبرندهُ عصبى ' نقص

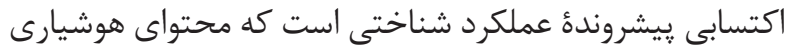

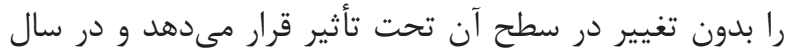

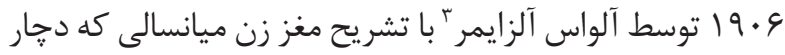

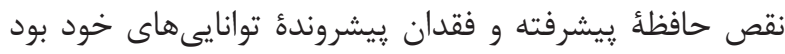

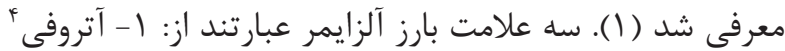

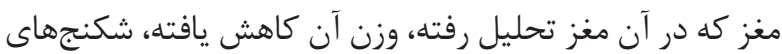

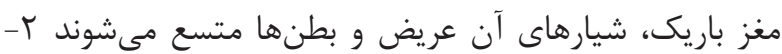

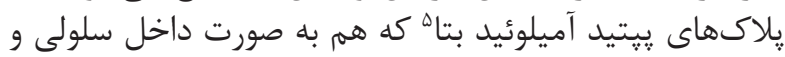

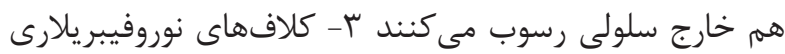
(NTF)

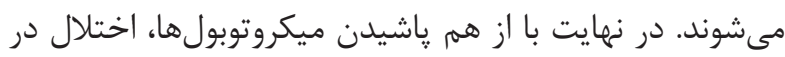

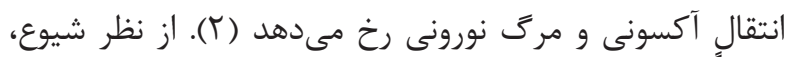

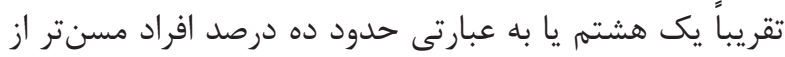

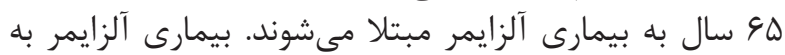

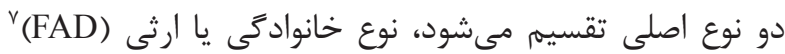

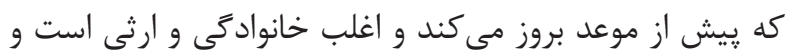

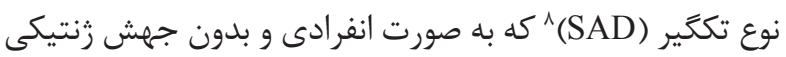

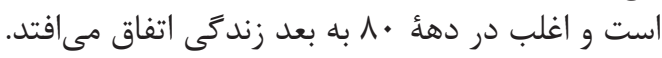

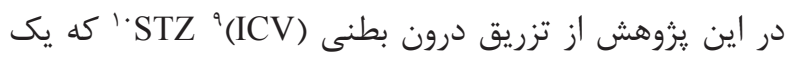

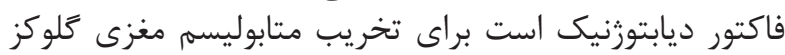

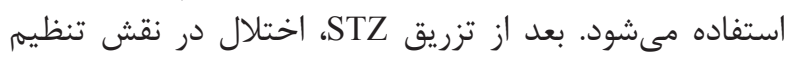

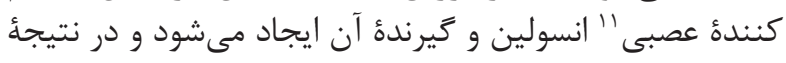

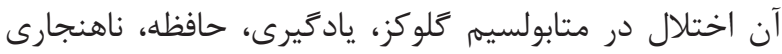

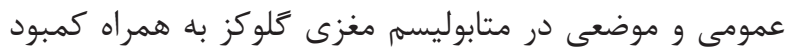

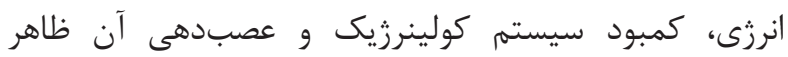

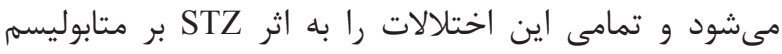

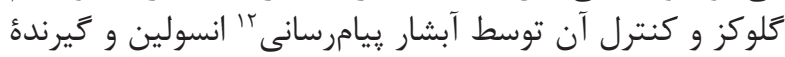

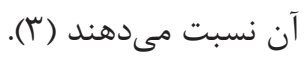

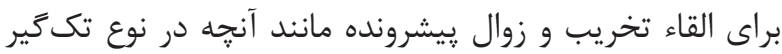

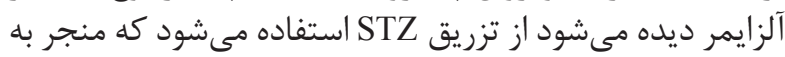

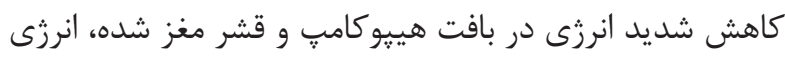

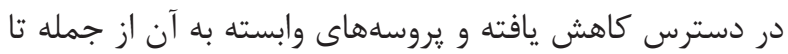

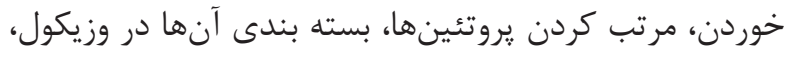

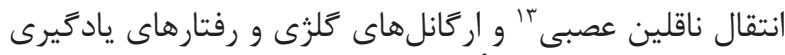

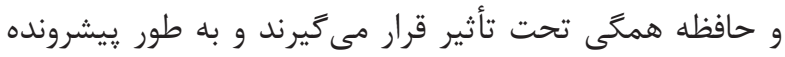

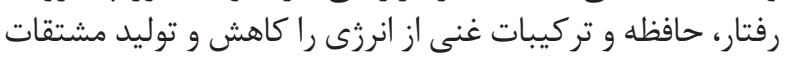

\footnotetext{
${ }^{12}$ Signaling

${ }^{13}$ Neurotransmitters

${ }^{14}$ Short-term memory

${ }^{15}$ Long-term memory

${ }^{16}$ Step wise manner

${ }^{17}$ Passive avoidance test

${ }^{18}$ Morris water maze

${ }^{19}$ Subventricular zone of lateral wall of lateral ventricle

${ }^{20}$ Sub-granular zone of dentate gyrus

${ }^{21}$ Hypothalamic sub-ependymal zone
} 
تعداد نوروسفرها در هر عكس به وسيلئ نرمافزار

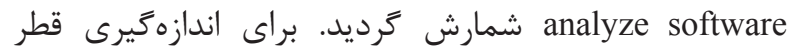

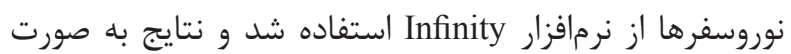

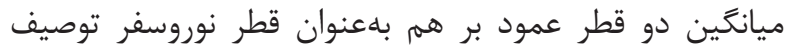

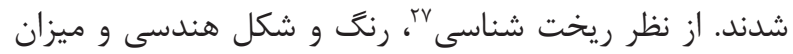

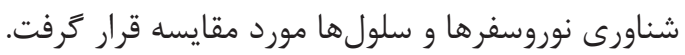

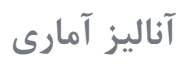

تمامى دادها به صورت ميانكَين \انحراف معيار كزارش شدهاند.

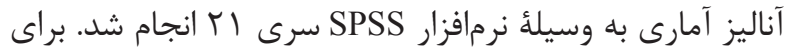

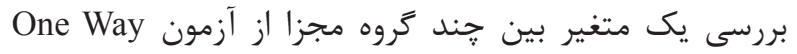
ANOVA بهنوان سطح معنى دارى درنظر كرفته شد.

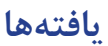

$$
\text { مشاهدات ريخت شناسى }
$$

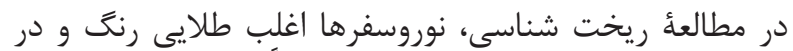

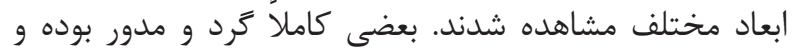

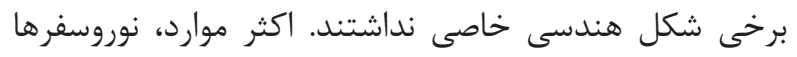

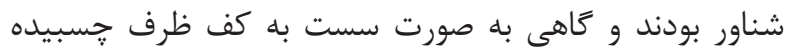

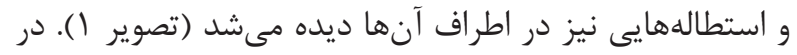

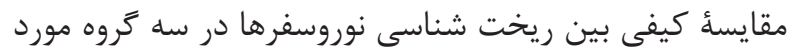

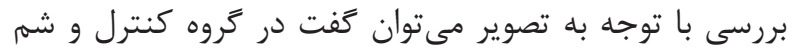

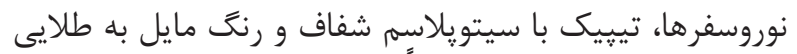

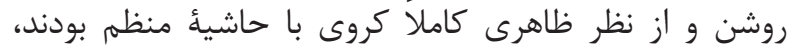

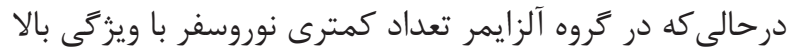

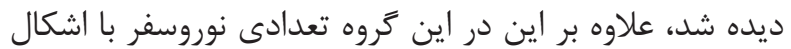

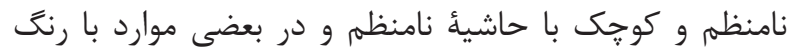

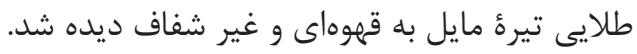

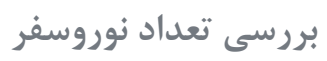

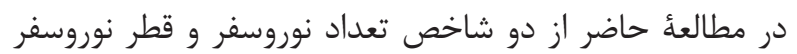

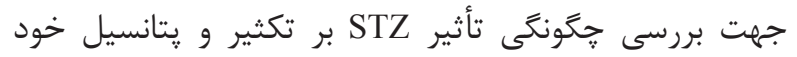

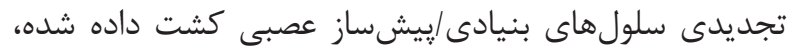

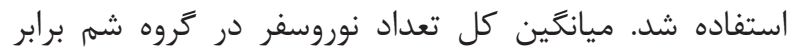
و TVT/T $\pm \Delta \&|\Lambda|$ د

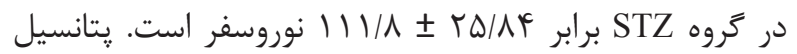

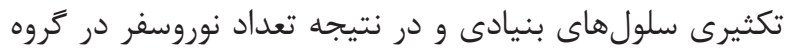

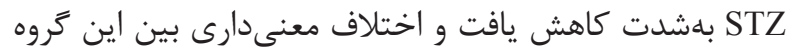

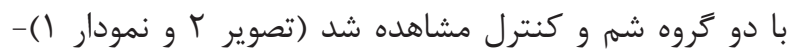

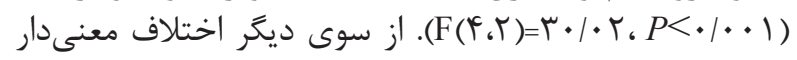

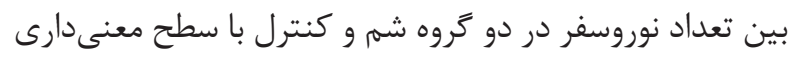
(1) كروه شم مشاهده شد.

\footnotetext{
${ }^{22}$ Rat

${ }^{23}$ Albino

${ }^{24}$ Wistar
}

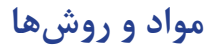

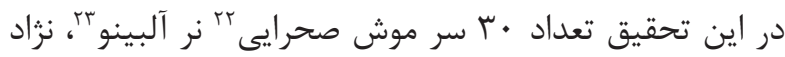

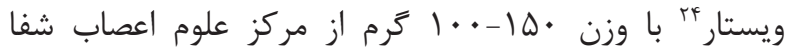

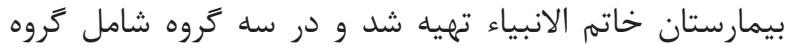

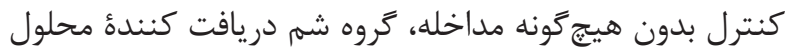

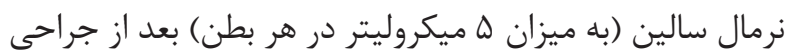

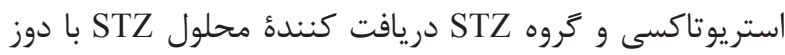

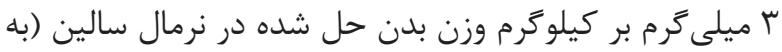

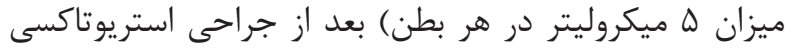

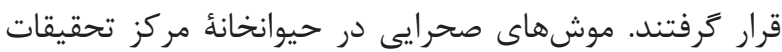

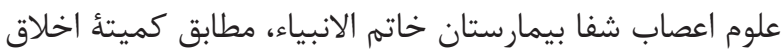

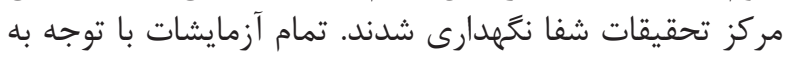

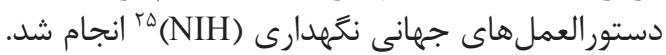

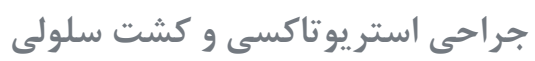

موشهاى صحرايى بيهوش شده و با استفاده از دستخاه

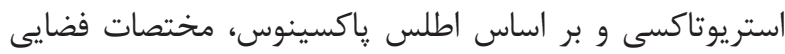

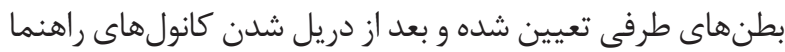

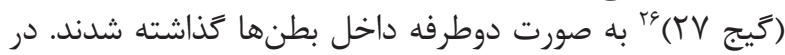

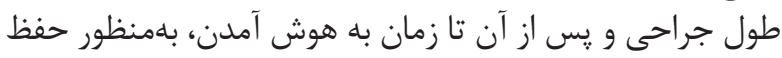

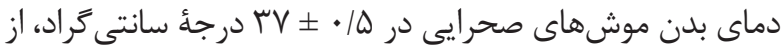

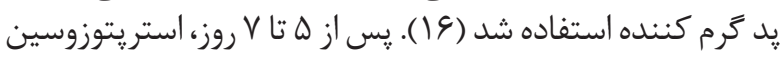

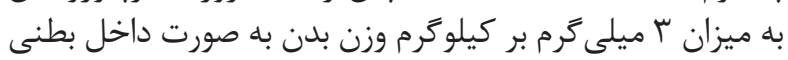

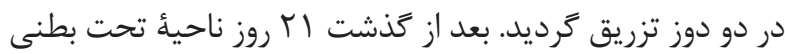

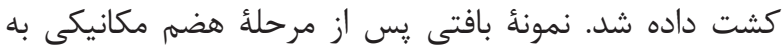

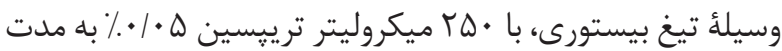

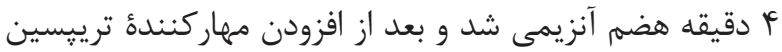

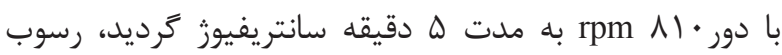

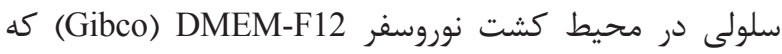

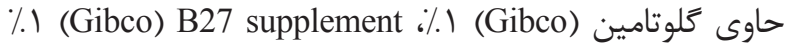

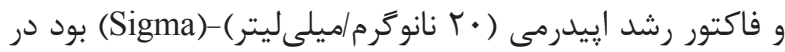

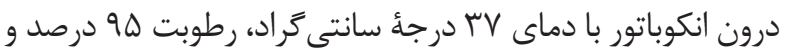
دى اكسيد كربن ه درصد كشت داده شدند.

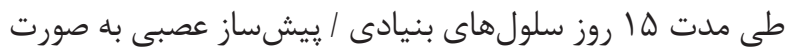

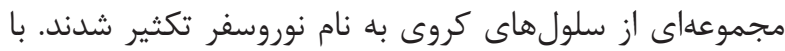

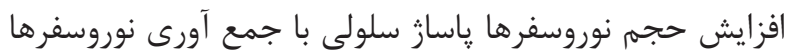

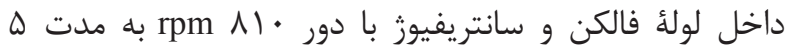

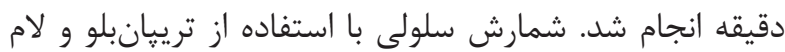

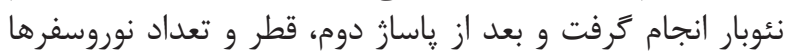

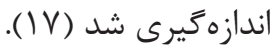

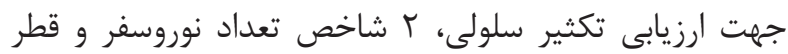

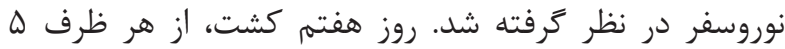

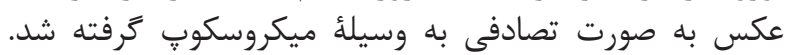

\footnotetext{
${ }^{25}$ National institutes of health

${ }^{26}$ 27-Gauge

${ }^{27}$ Morphology
} 


\section{ث}

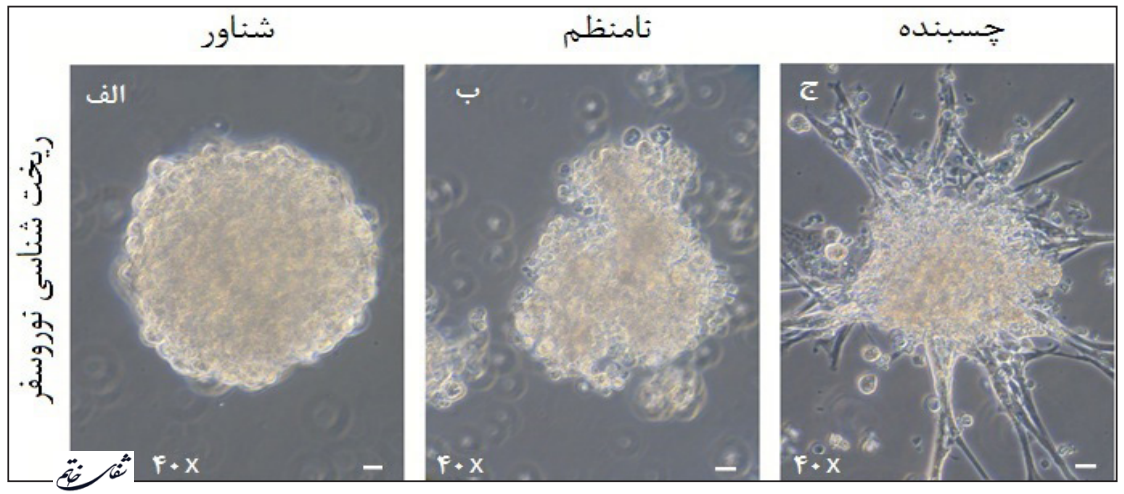

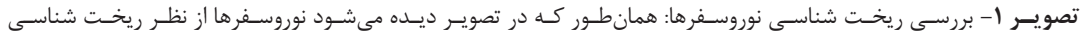

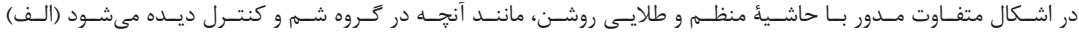

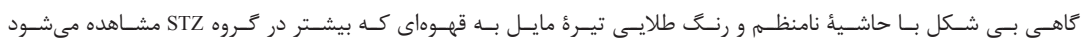

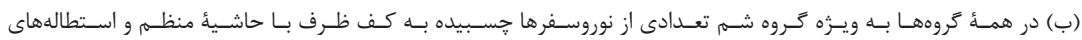

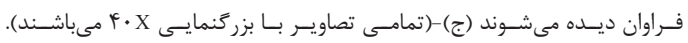

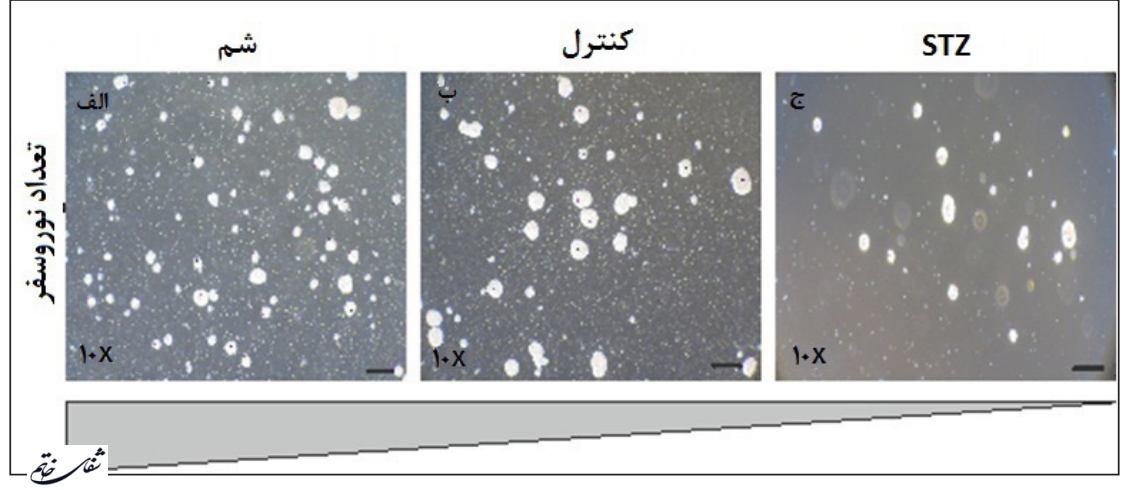

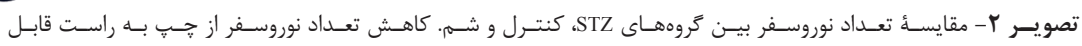

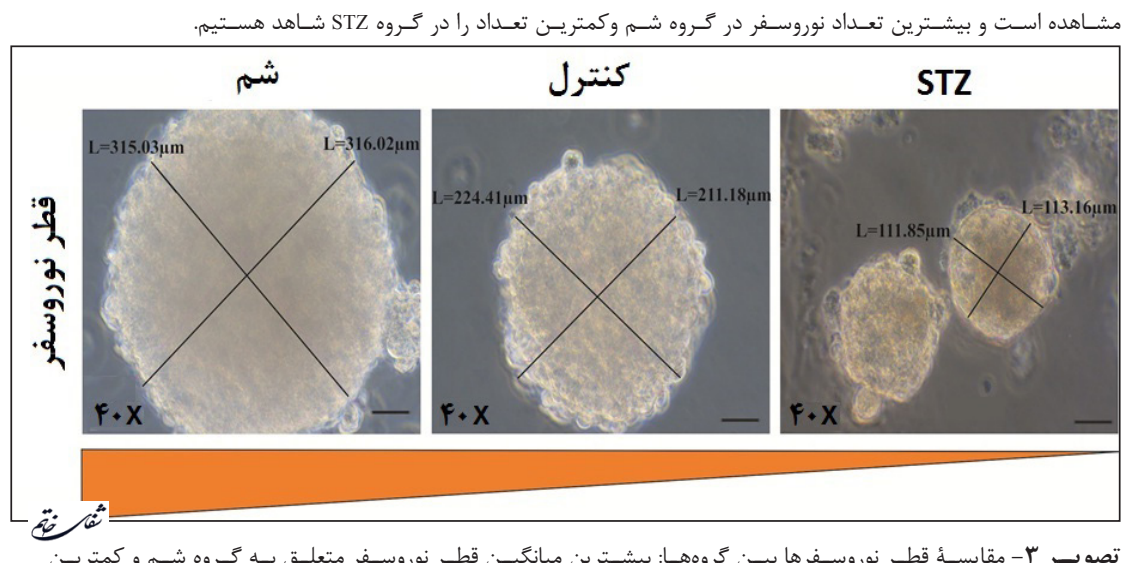

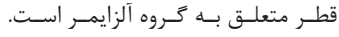
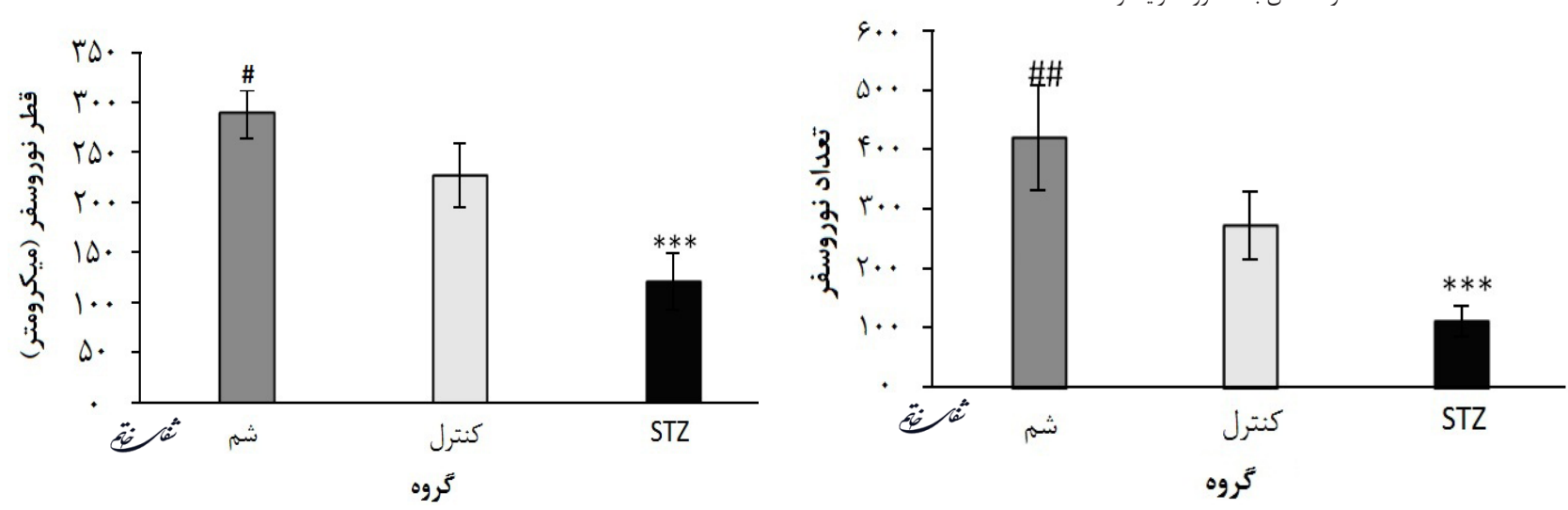

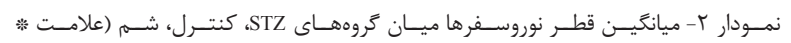

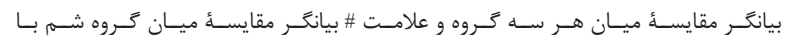

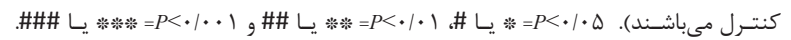

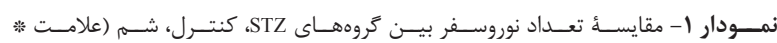

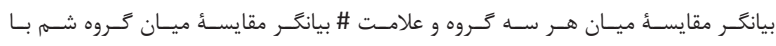

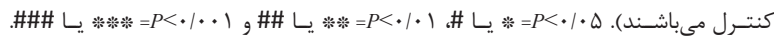




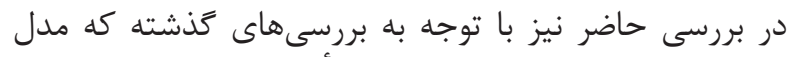

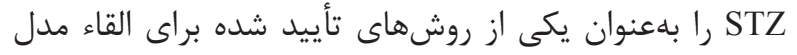

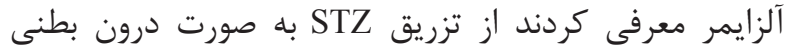

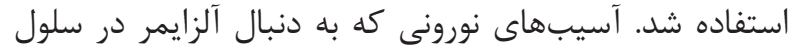

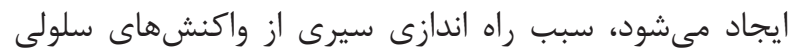

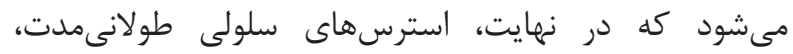

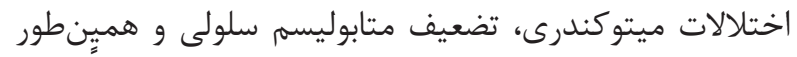

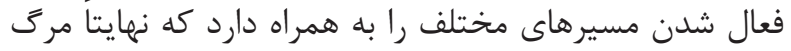

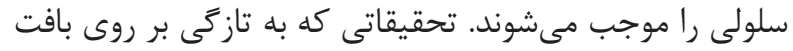

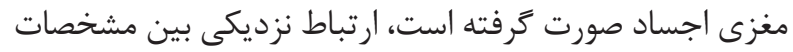

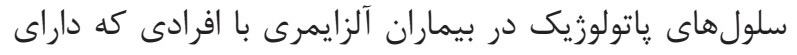

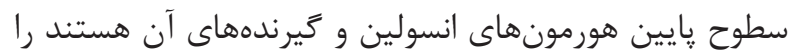

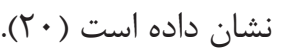

در اين مطالعه براى اولين بار تأثير STZ بر سلولهاى بنيادى

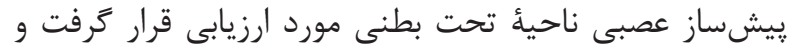

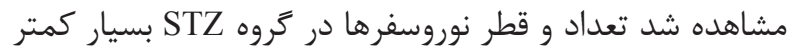

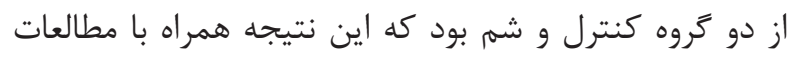

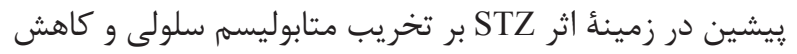

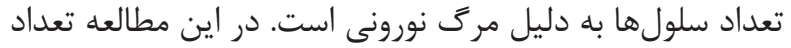

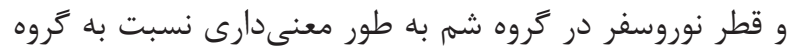

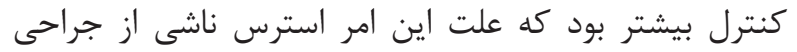

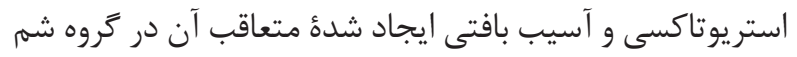

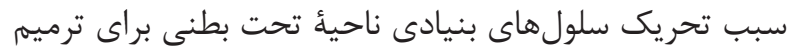

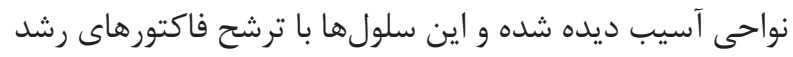

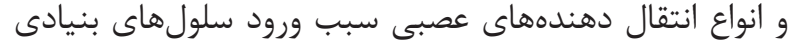
ناحيئ تحت بطنى به فاز تكثيرى مى شوند.

در اين روش، تركيب STZ به صورت داخل بطن مغزى

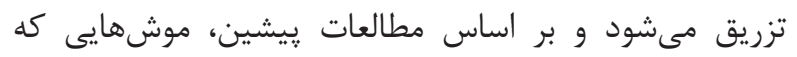

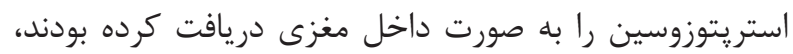

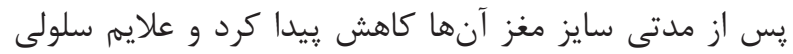

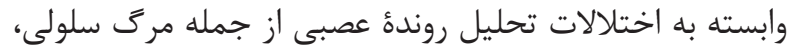

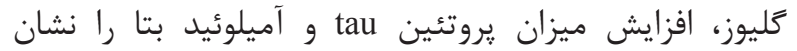

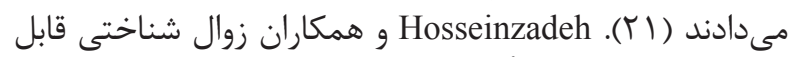

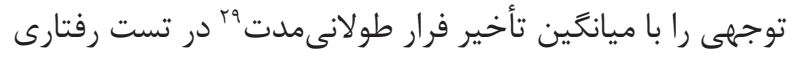

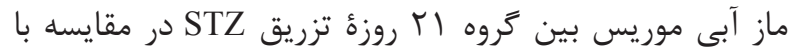

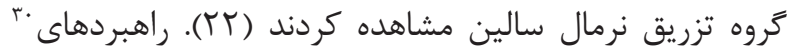

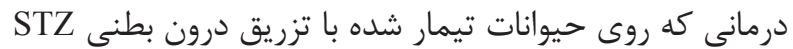

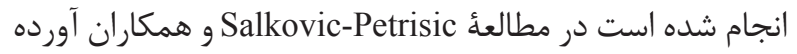

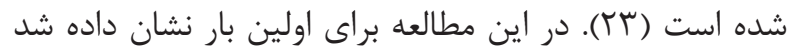

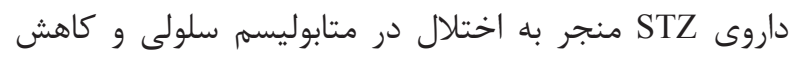

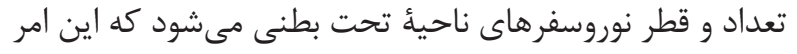

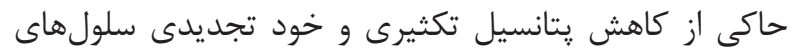
بنيادى إيشى از كاهن عصبى اين ناحيه است.
بر بسى قطر نوروسفر

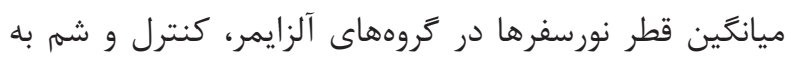

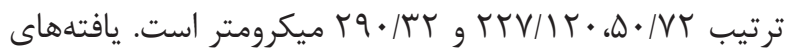

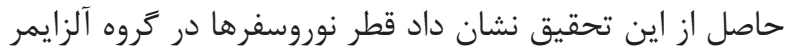

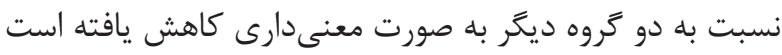

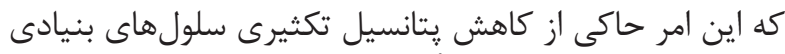

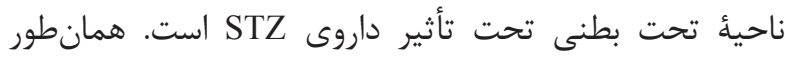

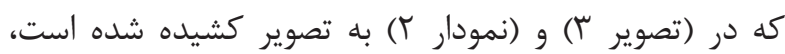

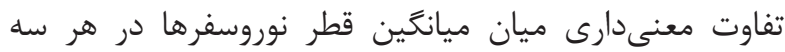

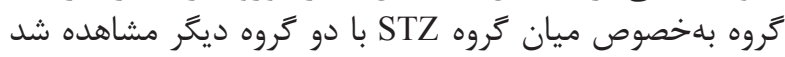

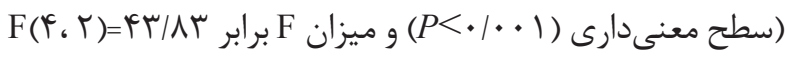

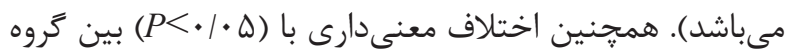

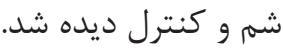

بحث و نتيجهَ ميرى

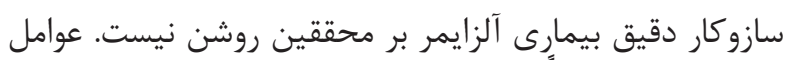

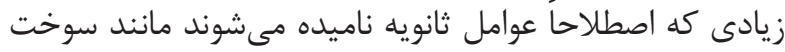

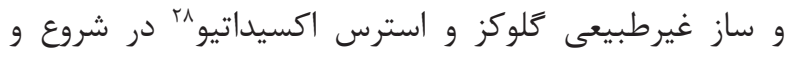

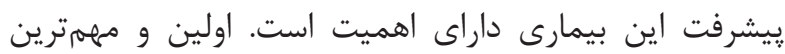

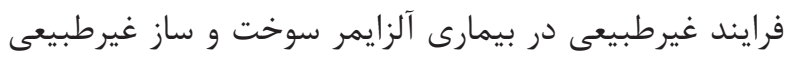

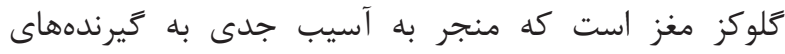

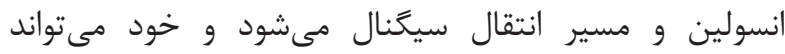

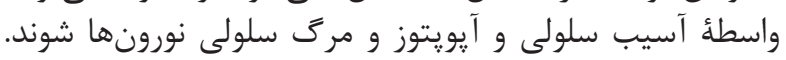

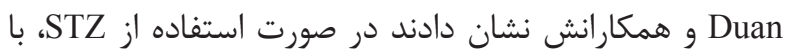

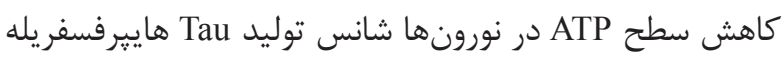

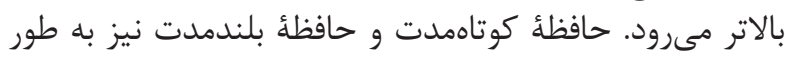

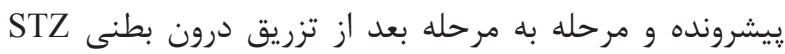

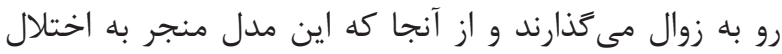

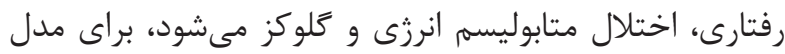
تكگير آلزايمر بسيار مناسب است (ه) (ه).

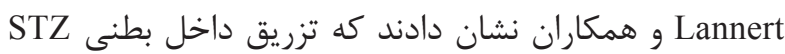

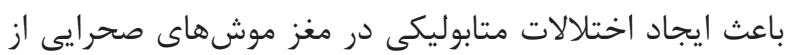

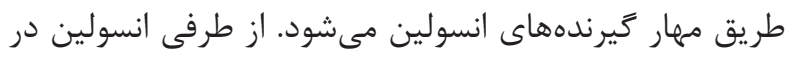

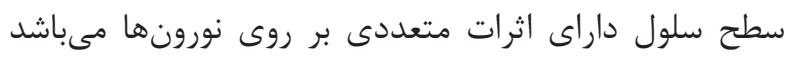

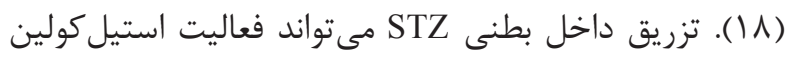

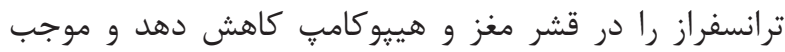

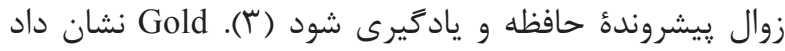

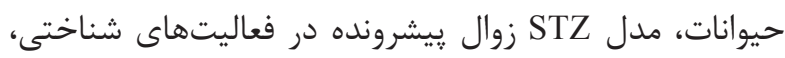

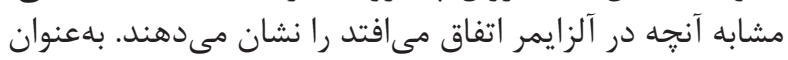

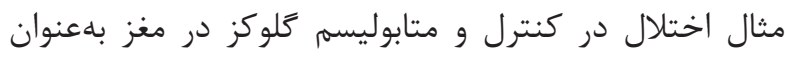

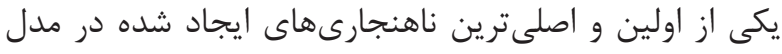
تكگير آلزايمر است و موجب المتين التلال در عملكرد انسولين

مىشود (19).

\footnotetext{
${ }^{28}$ Oxidative stress

${ }^{29}$ Prolonged mean scape latency

${ }^{30}$ Strategies
} 
1. Katzman R. The prevalence and malignancy of Alzheimer disease: a major killer. Arch Neurol. 1976; 33(4): 217-8.

2. Kandel ER, Schwartz JH, Jessell TM. Principles of neural science. 4th ed. New York: McGraw-Hill. 2000.

3. Peri A, Serio M. Neuroprotective effects of the Alzheimer's disease-related gene seladin-1. J Mol Endocrinol. 2008; 41(5): 251-61.

4. Guigon CJ, Zhao Li, Changxue Lu, Willingham MC, Cheng SY. Regulation of $\beta$-catenin by a novel nongenomic action of thyroid hormone $\beta$ receptor. Mol Cell Biol. 2008; 28(14): 4598-608.

5. Duan X, Kang E, Liu CY, Ming GL, Song H. Development of neural stem cell in the adult brain. Curr Opin Neurobiol. 2008; 18(1): 108-15.

6. Temple S. The development of neural stem cells. Nature. 2001; 414(6859): 112-7.

7. Alipour F, Karimzadeh F, Hasanzadeh G. P59: Triazine improved hippocampal injuries in animal model of Alzheimer's disease. The Neurosci J Shefaye Khatam. 2015; 2(4): 109.

8. Alvarez-Buylla A, Temple S. Stem cells in the developing and adult nervous system. J Neurobiol. 1998; 36(2): 105-10.

9. Eriksson PS, Perfilieva E, Bjork-Eriksson T, Alborn AM, Nordborg C, Peterson DA, et al. Neurogenesis in the adult human hippocampus. Nat Med. 1998; 4(11): 1313-7.

10. Galli R, Gritti A, Bonfanti L, Vescovi AL. Neural stem cells an overview. Circ Res. 2003; 92(6): 598-608.

11. Reynolds BA. Weiss S. Generation of neurons and astrocytes from isolated cells of the adult mammalian central nervous system. Science. 1992; 255(5052): 1707-10.

12. Allen E. The cessation of mitosis in the central nervous system of the albino rat. Waverley Press. 1912; p. 22.

13. Altman J, Bayer SA. Atlas of prenatal rat brain development. CRC Press. 1994.
14. Doetsch F, Caille I, Lim DA, Garcia-Verdugo JM, Alvarez-Buylla A. Subventricular zone astrocytes are neural stem cells in the adult mammalian brain. Cell. 1999; 97(6): 703-16.

15. Fuentealba LC, Obernier K, Alvarez-Buylla A. Adult neural stem cells bridge their niche. Cell Stem Cell. 2012; 10(6): 698-708.

16. Mohammadzadeh E, Alipour F, Khallaghi B. Evaluation of spatial memory impairment after intracerebroventricular streptozocin injection in adult rats. The Neurosci J Shefaye Khatam, 2014; 2(1): 40-5.

17. Reynolds BA, Rietze RL. Neural stem cells and neurospheres-re-evaluating the relationship. Nat Methods. 2005; 2(5): 333-6.

18. Lannert H, Hoyer S. Intracerebroventricular administration of Streptozocin causes long-term diminutions in learning and memory abilities and in cerebral energy metabolism in adult rats. Behav Neurosci. 1998; 112(5): 1199-208.

19. Gold PE. Role of glucose in regulating the brain and cognition. The American J Clinical Nutrition, 1995; 61(4): 987S-95S.

20. Cuevas E, Auso E, Telefont M, Morreale de Escobar G, Sotelo C, Berbel P, et al. Transient maternal hypothyroxinemia at onset of corticogenesis alters tangential migration of medial ganglionic eminencederived neurons. Eur J Neurosci. 2005; 22(3): 541-51.

21. Hassanzadeh G, Hosseini A, Pasbakhsh P, Akbari M, Ghaffarpour M, Takzare N, et al. Trimetazidine prevents oxidative changes induced in a rat model of sporadic type of Alzheimer s disease. Acta Med Iran. 2015; 53(1): $17-24$.

22. Hosseinzadeh S, Zahmatkesh $\mathrm{M}$, Heidari M, Hassanzadeh GR, Karimian M, Sarrafnejad A, et al. Hippocampal DHCR24 down regulation in a rat model of Streptozocin-induced cognitive decline. Neurosci Lett. 2015; 587: 107-12.

23. Salkovic-Petrisic M, Knezovic A, Hoyer S, Riederer P. What have we learned from the Streptozocin-induced animal model of sporadic Alzheimer's disease, about the therapeutic strategies in Alzheimer's research. J Neural Transm (Vienna). 2013; 120(1): 233-52. 